\title{
Research Domain Criteria: Cutting Edge Neuroscience or Galen's Humors Revisited?
}

\author{
Christopher A. Ross ${ }^{\mathrm{a}-\mathrm{e}}$ Russell L. Margolis ${ }^{\mathrm{a}} \mathrm{b}, \mathrm{e}$ \\ ${ }^{a}$ Division of Neurobiology, Department of Psychiatry and Behavioral Sciences, Johns Hopkins University \\ School of Medicine, Baltimore, MD, USA; ${ }^{b}$ Department of Neurology, Johns Hopkins University School of \\ Medicine, Baltimore, MD, USA; ' Department of Neuroscience, Johns Hopkins University School of Medicine, \\ Baltimore, MD, USA; ${ }^{d}$ Department of Pharmacology, Johns Hopkins University School of Medicine,

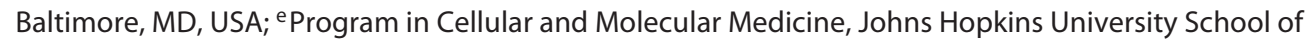 \\ Medicine, Baltimore, MD, USA
}

\section{Keywords}

Research Domain Criteria - Dimension - Category ·

Nosology $\cdot$ Humors

\begin{abstract}
The Research Domain Criteria (RDoC) scheme has guided the research agenda of the National Institute of Mental Health for the past decade. The essence of RDoC is its dimensional conception of mental illness, with the assumption that psychopathology is a manifestation of extremes along axes of neuropsychological variation. Research, it follows, should emphasize normal neuropsychological function and its associated neurocircuitry. We argue that RDoC, dressed in terms of modern neurobiology, is in fact a return to the humoral theory of Galen, a dimensional approach in which physical and mental health requires a balance of the four basic bodily humors (blood, black bile, yellow bile, and phlegm). The RDoC/Galenic approach may be useful in understanding those conditions best understood as extremes along a continuum, such as personality disorders. However, we contend that for the most severe psychiatric disorders categorically defined diseases such as schizophrenia, bipolar
\end{abstract}

\section{KARGER}

() 2018 S. Karger AG, Basel

E-Mail karger@karger.com

www.karger.com/mnp disorder, and autism - RDoC's Galenic dimensionalism is a retreat from the biomedical approach that seeks to find rational therapeutic targets by identifying etiologic factors and pathogenic pathways. Abandoning this medical model now, in the context of remarkable advances in genetics, neuroimaging, and neuroscience, is a major setback for the advancement of scientific psychiatry.

ㄷ) 2018 S. Karger AG, Basel

The Research Domain Criteria (RDoC) paradigm, a part of the US National Institute of Mental Health (NIMH) Strategic plan since 2008, was launched as a novel approach to psychiatric illness [1] in the context of frustration with progress in psychiatric research [2], and remains an essential component of the NIMH research strategy [3]. The goal is broad: " $\mathrm{RDoC}$ is nothing less than a plan to transform clinical practice by bringing a new generation of research to inform how we diagnose and treat mental disorders" [4]. While not yet extensively used in clinical practice, $\mathrm{RDoC}$ is becoming part of psychiatric training $[5,6]$, with the anticipation that it will guide practice in the future. 
However, we contend that, as applied to major mental illness, the scheme is conceptually flawed, and that the implementation of $\mathrm{RDoC}$ in psychiatric research, practice, and education is misplaced and a potential setback to our field. As applied to the most serious mental disorders, rather than reflecting a new and advanced approach to the science of mental illness, RDoC actually is a regression to a scheme reminiscent of the Galenic conceptualization of the four humors, which held a grip on medicine for nearly two millennia.

We have previously elaborated a set of critiques in which we demonstrate the inadequacies of $\mathrm{RDoC}$ compared to the disease model of major mental illness (Ross and Margolis, unpublished). In brief, we argue that as a top-down dimensional approach based on the study of normal function, $\mathrm{RDoC}$ deflects research away from a bottom-up approach to the etiologic and pathogenic processes most likely to yield mechanistic insight, nosological clarity, and novel therapeutic targets for the most serious mental illnesses. In one analogy, the $\mathrm{RDoC}$ approach to severe mental illness is reminiscent of trying to understand pneumonia by studying the biology of normal respiratory function - an interesting and potentially useful endeavor, but removed from the more immediately relevant issues of the etiology and pathogenesis of infectious agents and inflammatory processes.

A critical element of the RDoC scheme is that it "incorporates a dimensional approach to psychopathology" [7], exploring, as described in the NIMH 2008 Strategic Plan, "the full range of variation, from normal to abnormal ... to improve understanding of what is typical versus pathological" [8]. The new emphasis is on understanding normal neuropsychological function, emphasizing brain circuitry, with the assumption that psychopathology is the manifestation of extremes of normal neuropsychological function and hence extremes of normal brain circuitry function.

RDoC currently proposes five general domains of neuropsychological function, with constructs within each domain defining specific dimensional axes of function, undergirded by specific neural circuits [9]. We contend that this concept has merit in the exploration of psychiatric phenomena in which extremes of a trait can bring an individual to clinical attention - e.g., anxiety, mild forms of depression, or personality traits such as neuroticism or introversion. In such examples, the fundamental structure and operation of the brain remain intact, but because of differences along a continuum of normally distributed functions (cognition, emotion, or behavior), individuals towards the extremes may come to clinical attention.

Research Domain Criteria and Galen Revisited
However, RDoC attempts to apply this dimensional reasoning to the most severe forms of mental illness, including bipolar disorder, schizophrenia, autism, and the dementias. As an example: "... often both extremes of a dimension may be considered as 'abnormal' - for example, a complete lack of fear may be associated with aggressive or psychopathic behavior, and the opposite end of diminished reward-seeking may be mania" [9]. We contend that these disorders are best conceptualized not as extremes of normal, but rather according to the disease model, in which they are the result of etiologic and pathogenic processes that disturb the normal development and function of the brain and result in a disease with a distinct natural history. Even accepting the current RDoC domains and constructs as valid descriptions of normal brain processes (a debated assumption [10-12]), the available clinical, genetic, and imaging data suggest distinct differences between disease and normal states (e.g., in schizophrenia $[12,13])$. Psychiatric diseases, like other diseases in medicine, represent a disruption of normal neuropsychological and brain function, a break from normal.

The rationale for $\mathrm{RDoC}$ includes the argument that inadequacies in the DSM (and similarly, ICD) diagnostic systems imply the failure of any approach that views diseases as categorically distinct from normal states. There is indeed widespread recognition, including from the Chair of the DSM-5 Task Force [14], that DSM does not provide the final word in psychiatric nosology, with critiques of both its specific diagnostic categories [15-17] and its broader conceptual approaches $[15,18,19]$. By using the ill-defined term "disorder" [18] for every syndrome, DSM conflates entities that may fit the RDoC approach - e.g., disorders of personality that can be defined using a dimensional perspective - with disorders that should best be considered from the perspective of the disease model [20]. This confusion allows $\mathrm{RDoC}$ proponents to use DSM as a straw man in advocating an approach distinct from the disease model for even severe mental illness. However, the weakness of the DSM nosology does not invalidate the general concept that major mental illnesses are best defined using the disease model and thus are best viewed as categorically distinct from normal states of brain, emotion, and cognition.

In the absence of understanding based on etiology and pathogenesis, psychiatry has long had a tendency to resort to dimensional explanations. The origins are from Greek medicine. The central dimensional view of human personality and disease was the Hippocratic theory of four humors, promulgated and elaborated by Galen, Avicenna, and others in late antiquity and into the middle

Mol Neuropsychiatry 2018;4:158-163 159 
ages. Humoral theory gained ascendency at a time when even basic anatomical structures were poorly understood. As an all-encompassing system, it could be used to account for any condition. The four humors of Hippocrates

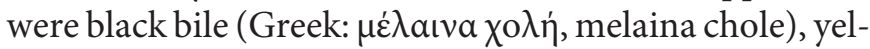
low bile (Greek: $\chi 0 \lambda \eta \dot{n}$, chole), phlegm (Greek: $\varphi \lambda \dot{\varepsilon} \gamma \mu \alpha$, phlegma), and blood (Greek: aĩ $\mu \alpha$, haima). One of the treatises attributed to Hippocrates, On the Nature of Man [21], describes the theory as follows:

The human body contains blood, phlegm, yellow bile and black bile. These are the things that make up its constitution and cause its pains and health. Health is primarily that state in which these constituent substances are in the correct proportion to each other, both in strength and quantity, and are well mixed. Pain occurs when one of the substances presents either a deficiency or an excess, or is separated in the body and not mixed with others.

The four humors corresponded to four traditional temperaments or personality types: sanguine (optimistic and social), choleric (irritable, excitable, or angry), melancholic (moody, sad, or quiet), and phlegmatic (calm and patient). Illness was believed to result when the humors were out of balance and would be treated by attempting to restore balance, for instance with emetics or bleeding. Thus, disease was viewed as an extreme of a personality type with a speculative pathophysiology (excess or deficiency of one of the humors, or more generally imbalance of the humors). Treatment of illness (both psychiatric and nonpsychiatric) would be accomplished by bringing the hypothesized pathophysiology - the humors - back in balance (Fig. 1).

One strand of Galenic reasoning, that personality types can be understood by considering traits along dimensional axes, continued into modern times, forming the basis of the theoretical systems of such prominent philosophers, physiologists, and psychologists as Kant $[22,23]$, Pavlov [23], Wundt [23], and Jung [24]. In particular, Eysenk and others described two dimensions of personality - introversion-extraversion and neuroticismstability - that defined four general personality types remarkably similar to those of Galen, to the extent that Eysenk specifically compared his system to that of Galen $[22,25]$. More recently, the five-factor personality model, an elaboration of the same concept, has gained widespread acceptance [26]. While these models have proven quite successful in understanding personality and individual vulnerabilities, they do not elucidate the most severe forms of mental illness.

The extension of humoral theory from personality traits to more severe forms of mental illness began with Galen himself:

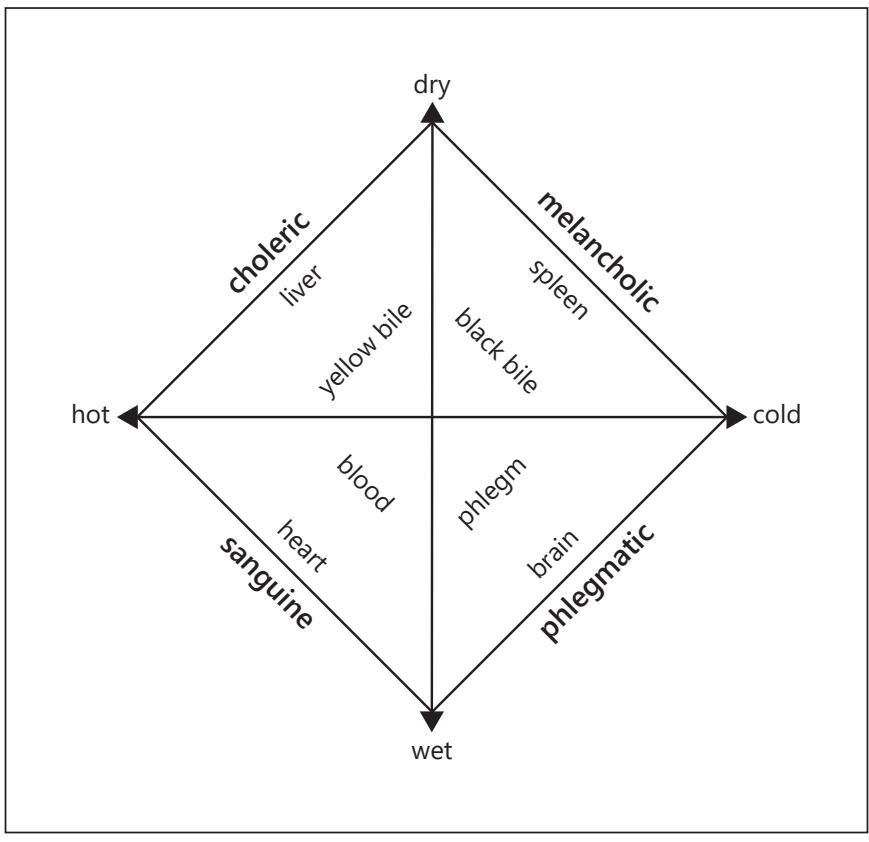

Fig. 1. The four humors and dimensional reasoning. The figure depicts a classic version of the four humors (the fundamental fluids of the body: blood, yellow bile, black bile, and phlegm), with the organ to which each was most closely associated and the personality type corresponding to each humor. Health, physical and mental, was a consequence of a balance of the humors. The two axes, wet-dry and hot-cold, describe the essential qualities of each humor. Each humor was also identified with an element (air, fire, earth, and water). Various philosophers and physicians over the centuries substantially elaborated and modified this basic theory.

I know a man from Cappadocia, who had gotten a nonsensical thing into his head and because of this declined into melancholy ... His friends saw him weeping and asked him about his grief. At that, he sighed deeply and answered, saying that he was worried that the person, of whom the poets relate ... carries the world and is called Atlas, would become tired because he had carried it for so long. Thus there was a danger the sky would fall on the earth and smash it. [27]

Galen interpreted such a melancholic (and quite possibly psychotic) condition to be the consequence of excess black bile, which could accumulate in the brain and cause a variety of psychopathologies, and which should be treated by purging [28].

We suggest that the RDoC scheme perpetuates the kind of dimensional thinking about disease prominent in Galenic thought and largely rejected by the biomedical scientists of the Enlightenment [19]. Indeed, the structure of $\mathrm{RDoC}$, usually displayed as a matrix, can also be configured in the same fashion as the four humors, a visual de- 


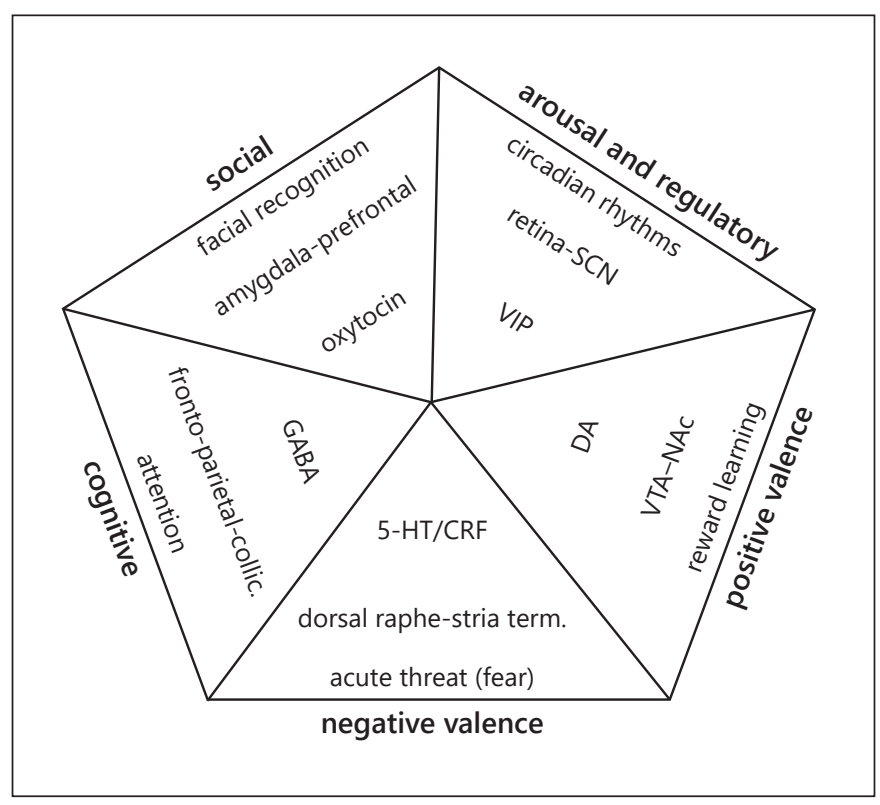

Fig. 2. Formal congruence of National Institute of Mental Health RDoC and the Galenic four humors. Depicted: RDoC domains (external labels, in bold), a construct proposed to underlie each domain (base of each triangle), a proposed neurocircuit for the construct (midtriangle), and a neurotransmitter relevant to the neurocircuit (tip of each triangle) [32-37]. As in the four humors, it is proposed that mental health requires a balance of the factors underlying each domain. Note the congruence of the overall formal structure with that of Figure 1. Other constructs, and different aspects of proposed underlying neurocircuitry, have been hypothesized for each domain, but the specific details do not alter the formal structure - balance across the constructs and circuitry is required for mental health. 5-HT, serotonin; collic., collicular; $\mathrm{CRF}$, corticotropin-releasing factor; DA, dopamine; GABA, gamma-aminobutyric acid; NAc, nucleus accumbens; RDoC, Research Domain Criteria; SCN, suprachiasmatic nucleus; stria term., stria terminalis; VIP, vasoactive intestinal peptide; VTA, ventral tegmental area.

piction of the formal congruence of the two systems (Fig. 2). Instead of humors, we now have the five RDoC domains with their associated constructs. Similar to humoral theory, there are a set of hypothesized physiological substrates to each of the domains and constructs. While the neurobiology underlying the $\mathrm{RDoC}$ constructs is certainly far more sophisticated than the humors, it is notable that the focus is on well-known neurotransmitters and circuits derived from the study of psychiatric drug pharmacology and normal physiology rather than the nascent etiology and pathogenesis of major mental illness.

Confounding humoral dimensions with categorically defined disease may unfortunately have serious consequences. Cancer was believed by Galen and his followers

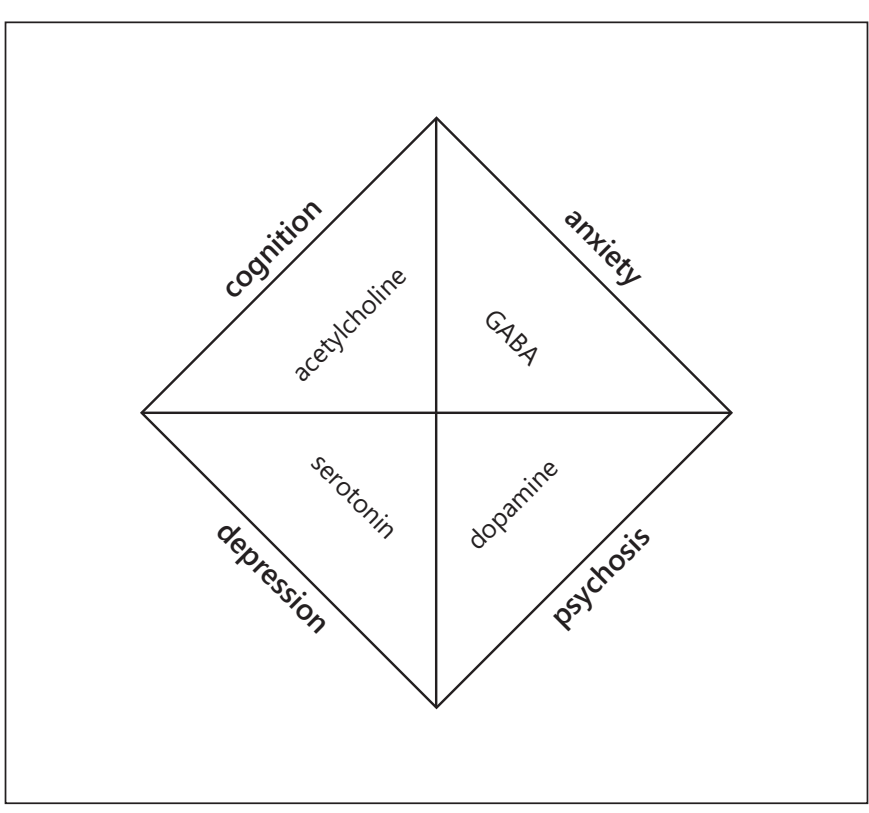

Fig. 3. "Chemical imbalances of the brain" and the four humors. As a simplification, a depiction of four of the most prominent types of psychopathology with a neurotransmitter often associated with each. As in the RDoC and the four humors schemes, proper mental health is conceptualized dimensionally, as a balance of the factors underlying each type of symptom. GABA, gamma-aminobutyric acid; RDoC, Research Domain Criteria.

to arise from an excess of black bile and to afflict those with a melancholic disposition [29]. In the 16th century, Vesalius searched for black bile, but could not find it. Progress in oncology only emerged after Virchow, in the mid-19th century, had first proposed the cellular concept of neoplasia, replacing humoral theory and practice with a modern, and increasingly successful, approach to oncology. Psychiatry has struggled to make the same conceptual leap. For instance, over the past 50 years, with advances in neuroscience leading to theories of psychopathology based on neurotransmitters and their receptors, psychiatrists often inform patients that mental illness arises from a "chemical imbalance in the brain." This simplistic concept has been widely assimilated by both clinicians [30] and the public (e.g., wikiHow: "How to deal when you have a chemical imbalance" [31]). While serving as a potentially useful metaphor to remove stigma and self-blame from mental illness, this phrase implies a dimensional view of psychopathology: everyone has the same set of neurotransmitters, they are normally in balance with each other, and mental illness arises when they go out of balance (Fig. 3). One consequence has been attempts to treat individual symptoms by using drugs that 
supposedly have some specificity in altering the balance of neurotransmitters or the process of neurotransmission, a response that Ghaemi points out results in "extremist individualization" and a reversion to the nonscientific aspects of humorism which "produced 2000 years of dehumanizing, harmful bleeding and purging” [20].

There is no question that our understanding of psychiatric conditions remains in its infancy, and that research into the mechanisms of normal emotion, cognition, and behavior emphasized by the RDoC initiative has value. This endeavor is intrinsically of interest and likely to be especially useful in understanding the bases of psychiatric problems that are either extremes of normal phenomena or debilitating reactions to extreme circumstances, including personality disorders, anxiety disorders, "adjustment disorders," and posttraumatic stress disorder. Our objection is to the assumption that $\mathrm{RDoC}$, with its dimensional logic, provides the best approach to research into those psychiatric disorders best viewed using the disease model.

We argue here that the $\mathrm{RDoC}$ scheme, while dressed up in the language of contemporary neurobiology, actually preserves the same formal structure as the old Galenic humoral paradigms. This is ironic, as the power of contemporary genetics, epidemiology, and neuroscience has made it possible to delineate specific causes and rational treatments for Mendelian genetic diseases (e.g., Huntington's disease) and diseases with a known pathophysiology (e.g., Addison's disease, alcohol withdrawal, and syphilis). Furthermore, at long last, this biomedical approach using the disease model is enabling us to address fundamental questions about the etiology and pathogenesis of the categorically defined disorders with complex etiologies. Abandoning the disease model now for the sake of a system that does not appreciate the need to describe disease as separate from wellness, and shifts focus away from etiology, pathogenesis, natural history, and rational therapeutics, is a setback for a truly scientific psychiatry.

\section{Acknowledgments}

The authors thank the participants at the Molecular Psychiatry and Translational Psychiatry 2016 meetings for feedback on some initial conceptualizations of these ideas presented in 2016. Steven McCarroll presented a slide entitled "the amorphous brain," similar to Figure 3 in this paper, at the 2016 Molecular Psychiatry meeting.

\section{Disclosure Statement}

Dr. Ross reports grant support from NIH and CHDI, previous support from JNJ/Janssen, Teva, and Raptor/Horizon, and consulting for Teva, uniQure, Roche/Ionis, and HSG. Dr. Margolis reports grant support from NIH, ABCD Charitable Trust, and Teva.

\section{Funding Sources}

This work was supported in part by the ABCD Charitable Trust, which had no role in the conceptualization, preparation, or review of the manuscript.

\section{References}

1 Insel T, Cuthbert B, Garvey M, Heinssen R, Pine DS, Quinn K, et al. Research domain criteria (RDoC): toward a new classification framework for research on mental disorders. Am J Psychiatry. 2010 Jul;167(7):748-51.

2 Morris SE, Cuthbert BN. Research Domain Criteria: cognitive systems, neural circuits, and dimensions of behavior. Dialogues Clin Neurosci. 2012 Mar;14(1):29-37.

3 National Institute of Mental Health: National Institute of Mental Health: A Strategic Plan for Research. 2015.

4 Insel T. Transforming diagnosis. 2013. www. nimh.nih.gov/about/directors/thomas-insel/ blog/2013/transforming-diagnosis.shtml.
5 Muzyk AJ, Gagliardi JP, Rakesh G, Jiroutek MR, Radhakrishnan R, Pae CU, et al. Development of a Diverse Learning Experience for Diverse Psychiatry Resident Needs: A FourYear Biological Psychiatry Curriculum Incorporating Principles of Neurobiology, Psychopharmacology, and Evidence-Based Practice. Psychiatry Investig. 2017 May; 14(3):289-97.

6 Benjamin S. A DSM-5 to-do list for adult psychiatry residency directors. Acad Psychiatry. 2014 Feb;38(1):67-71.

7 Cuthbert BN. Translating intermediate phenotypes to psychopathology: the NIMH Research Domain Criteria. Psychophysiology. 2014 Dec;51(12):1205-6.

8 US National Institute of Mental Health. Strategy 1.4 of the 2008 NIMH Strategic Plan; 2008:1-60.
9 Cuthbert BN, Insel TR. Toward the future of psychiatric diagnosis: the seven pillars of RDoC. BMC Med. 2013 May;11(1):126.

10 Rodebaugh TL, Scullin RB, Langer JK, Dixon DJ, Huppert JD, Bernstein A, et al. Unreliability as a threat to understanding psychopathology: the cautionary tale of attentional bias. J Abnorm Psychol. 2016 Aug;125(6):840-51.

11 Hess JL, Kawaguchi DM, Wagner KE, Faraone SV, Glatt SJ. The influence of genes on "positive valence systems" constructs: A systematic review. Am J Med Genet B Neuropsychiatr Genet. 2016 Jan;171B(1):92-110.

12 Birnbaum R, Weinberger DR. Genetic insights into the neurodevelopmental origins of schizophrenia. Nat Rev Neurosci. 2017 Dec; 18(12):727-40 
13 Piper M, Beneyto M, Burne TH, Eyles DW, Lewis DA, McGrath JJ. The neurodevelopmental hypothesis of schizophrenia: convergent clues from epidemiology and neuropathology. Psychiatr Clin North Am. 2012 Sep; 35(3):571-84.

14 Kupfer D. The DSM-5 - an interview with David Kupfer. BMC Med. 2013 Sep;11(1): 203.

15 Wakefield JC. The DSM-5 debate over the bereavement exclusion: psychiatric diagnosis and the future of empirically supported treatment. Clin Psychol Rev. 2013 Nov;33(7):82545.

16 Hoge CW, Yehuda R, Castro CA, McFarlane AC, Vermetten E, Jetly R, et al. Unintended consequences of changing the definition of posttraumatic stress disorder in DSM-5: critique and call for action. JAMA Psychiatry. 2016 Jul;73(7):750-2.

17 Parker G. The DSM-5 classification of mood disorders: some fallacies and fault lines. Acta Psychiatr Scand. 2014 Jun;129(6):404-9.

18 Jablensky A. The disease entity in psychiatry: fact or fiction? Epidemiol Psychiatr Sci. 2012 Sep;21(3):255-64.

19 Zachar P, Kendler KS. The Philosophy of Nosology. Annu Rev Clin Psychol. 2017 May; 13(1):49-71.
20 Ghaemi SN. Taking disease seriously in DSM. World Psychiatry. 2013 Oct;12(3):210-2.

21 Hippocrates of Cos. On the Nature of Man. In: Lloyd G, editor. Hippocratic Writings. London: Penguin Books; 1983. p. 383.

22 Stelmack RM, Stalikas A. Galen and the humour theory of temperament. Pers Individ Dif. 1991;12(3):255-63.

23 Ruch W. Pavlov's types of nervous system, Eysenck's typology and the Hippocrates-Galen temperaments: an empirical examination of the asserted correspondence of three temperament typologies. Pers Individ Dif. 1992; 13(12):1259-71.

24 Jung CG. Psychological Types. Princeton (New Jersey): Princeton University Press; 1971.

25 Eysenck HJ. Personality and Individual Differences: A Natural Sciences Approach. New York: Plenum Press; 1985.

26 Costa PT, McCrae RR. Personality in Adulthood: A Five-Factor Theory Perspective. New York: Taylor \& Francis; 2013.

27 Mattern SP. The Prince of Medicine: Galen in the Roman Empire. Oxford: Oxford University Press; 2013.

28 Mattern S. Galen and his patients. Lancet. 2011 Aug;378(9790):478-9.

29 Hajdu SI. Greco-Roman thought about cancer. Cancer. 2004 May; 100(10):2048-51.

30 Leo J, Lacasse J. The Media and the Chemical Imbalance Theory of Depression. Society. 2008;45(1):35-45.
31 Matsko CM: How to Deal When You Have a Chemical Imbalance. 2018.

32 Marcinkiewcz CA, Mazzone CM, D'Agostino G, Halladay LR, Hardaway JA, DiBerto JF, et al. Serotonin engages an anxiety and fear-promoting circuit in the extended amygdala. $\mathrm{Na}-$ ture. 2016 Sep;537(7618):97-101.

33 Hikida T, Morita M, Macpherson T. Neural mechanisms of the nucleus accumbens circuit in reward and aversive learning. Neurosci Res. 2016 Jul; 108:1-5.

34 Brancaccio M, Enoki R, Mazuski CN, Jones J, Evans JA, Azzi A. Network-mediated encoding of circadian time: the suprachiasmatic nucleus (SCN) from genes to neurons to circuits, and back. J Neurosci. 2014 Nov;34(46): 15192-9.

35 Fairhall SL, Ishai A. Effective connectivity within the distributed cortical network for face perception. Cereb Cortex. 2007 Oct 17(10):2400-6.

36 Westberg L, Henningsson S, Zettergren A, Svärd J, Hovey D, Lin T, et al. Variation in the Oxytocin Receptor Gene Is Associated with Face Recognition and its Neural Correlates. Front Behav Neurosci. 2016 Sep;10:178.

37 Womelsdorf T, Everling S. Long-Range Attention Networks: Circuit Motifs Underlying Endogenously Controlled Stimulus Selection. Trends Neurosci. 2015 Nov;38(11):682-700. 